Rodríguez-Pulido, F., Rodríguez-Pulido, J., Rodríguez-García, M.A. \& Bartolomé-Yumar, A.E. (2021). Formación no presencial: materia de grado de medicina, entorno virtual, pandemia y satisfacción. Revista Electrónica Interuniversitaria de Formación del Profesorado, 24(3), 57-71.

DOI: https://doi.org/10.6018/reifop.459591

\title{
Formación no presencial: materia de grado de medicina, entorno virtual, pandemia y satisfacción
}

\author{
Francisco Rodríguez Pulido ${ }^{(1)}$, Josefa Rodríguez Pulido ${ }^{(2)}$, María Angeles Rodríguez García ${ }^{(1)}$, \\ Alba Elisa Bartolomé Yumar ${ }^{(1)}$ \\ ${ }^{(1)}$ Universidad de La Laguna; ${ }^{(2)}$ Universidad de Las Palmas de Gran Canaria
}

\section{Resumen}

Esta investigación plantea conocer el grado de satisfacción que los estudiantes universitarios tienen respecto a la experiencia formativa no presencial de los procesos de enseñanzaaprendizaje en investigación en tiempos de pandemia. Para evaluarlo aplicamos un cuestionario, que hemos adaptado de unas escalas estandarizadas de satisfacción de alumnos universitarios con respecto a la formación online, CSAUF, (Monsalve-Gómez, Botero-Botero, y Montoya-Suárez, 2014) y semipresencial (Cabero-Almenara, Llorente y Puentes, 2010), a 64 (estudiantes) universitarios, elegidos al azar, de ambos géneros con edades comprendidas entre los 23 y más de 30 años que cursan el grado de medicina. Se emplea una metodología tipo cuantitativa con un diseño descriptivo y comparativo. Los resultados indican que los estudiantes de la muestra presentan un grado de satisfacción bastante favorable con la experiencia formativa, siendo el funcionamiento y los recursos didácticos del entorno virtual, así como el perfil del docente, las variables discriminativas mejor valoradas. No existen diferencias significativas (IC 99,95\%) por razón del género, edad y rotaciones realizadas durante las clases no presenciales. Los estudiantes universitarios valoran, de forma muy positiva, la experiencia vivida.

\section{Palabras clave}

satisfacción; formación no presencial; estudiantes de medicina; educación superior.

\section{Contacto:}

Francisco Rodríguez Pulido,fpulido15@me.com. 


\title{
Non-presential training: degree of medicine, virtual environment, pandemic and satisfaction
}

\begin{abstract}
This research proposes knowing the degree of satisfaction that university students have with respect to the non-face-to-face training experience of the teaching-learning processes in research in times of pandemic. To evaluate it, we applied a questionnaire, which we have adapted from some standardized scales of satisfaction of university students with respect to online training, CSAUF, (Monsalve-Gómez, Botero-Botero, and Montoya-Suárez, 2014) and blended (Cabero-Almenara, Llorente and Puentes, 2010), 64 university (students), chosen at random, of both genders with ages between 23 and more than 30 years who are studying the degree of medicine. A quantitative type methodology is used with a descriptive and comparative design. The results indicate that the students in the sample present a fairly favorable degree of satisfaction with the training experience, being the functioning and the didactic resources of the virtual environment, as well as the teacher's profile, the best valued discriminative variables. There are no significant differences $(99.95 \% \mathrm{Cl})$ due to gender, age and rotations carried out during non-contact classes. University students value, in a very positive way, the lived experience.
\end{abstract}

\section{Key words}

Satisfaction; non-face-to-face training; medicine students; higher education.

\section{Introducción}

La satisfacción del alumnado como constructo relevante para la carrera en universitarios, ni es discutible (Merino-Soto et al., 2017). La aplicación de escalas de valoración de la satisfacción del alumnado es un proceso previo para el desarrollo de estrategias de acción en la mejora de la calidad de la enseñanza universitaria (Cuso et al., 2015).

A nivel internacional la satisfacción académica ha sido ampliamente estudiada (Melo et al., 2015), a raíz de la pandemia, los estudios sobre la satisfacción del universitario en la formación no presencial son escasos.

La satisfacción del alumnado implica una relación con los programas de estudio, el sistema de evaluación, los recursos didácticos y la comunicación entre alumno y profesor (Robbins, 1998). Pineda-Alfonso y Piña (2020) la concepción de la materia responde a una visión de los aspectos científicos.

El contexto universitario demanda la necesidad de indagar en los aspectos que inciden en los procesos educativos no presenciales (De Agüero-Servín, 2020). Según Toledo (2020) en el escenario de pandemia Covid 19, el docente universitario de forma forzada asume un rol diferente e incorpora herramientas, sin mayor formación.

Ordorika (2020) el cambio de la docencia presencial ha generado situaciones dificultosas, de ahí, la necesidad de indagar en el desarrollo de los aprendizajes. Zerpa y Zerpa (2020) los desafíos de la transformación digital son: la formación de los docentes y la comunicación con el alumnado. Los planes formativos existentes en la institución superior reclaman una reorientación en la formación del profesorado. Podría ser un buen momento para que el 
profesorado de la institución superior reinvente sus procesos formativos, con el apoyo de las políticas programáticas establecidas por la gobernanza universitaria.

\section{Método}

\section{Objetivos e Hipótesis de la investigación}

Nos propusimos, en primer lugar, conocer el grado de satisfacción de los universitarios y comprobar si existen diferencias en el nivel de satisfacción de los estudiantes por razón del género, la edad y las rotaciones realizadas durante las clases no presenciales Averiguamos las diferencias estadísticamente significativas, a través de las siguientes hipótesis:

$H_{0}$ : No existen diferencias estadísticamente significativas en el grado de satisfacción de la formación en función del género, la edad y las rotaciones realizadas durante las clases no presenciales.

$H_{1}$ : Existen diferencias estadísticamente significativas en el grado de satisfacción de la formación en función del género, la edad y las rotaciones realizadas durante las clases no presenciales.

Metodología de corte cuantitativa, con un diseño descriptivo y comparativo para indagar en los fenómenos mediante la formulación de objetivos e hipótesis que brindan la posibilidad de comprobar y verificar lo deducido con la experiencia (Bunge, 2010) en situación natural (Kerlinger, 1979, p.116): “... en este tipo de investigaciones, no se construye ninguna situación, sino que se observan situaciones ya existentes, no provocadas intencionalmente por el investigador"; y la descripción de determinadas características de una población a partir de los datos obtenidos en una muestra relativamente pequeña de esa población, con la posibilidad de predecir el comportamiento de los sujetos a través de cuestionarios, cuyos resultados pueden ser generalizados y extrapolables (Hernández et al., 2010).

\section{Muestra}

Muestreo probabilístico aleatorio simple (Cochran y Bouclier, 1980, p.41), como informantes claves, según el número de componentes previamente definido (Cerón, 2006, p. 143). En este caso ( tabla 1), está constituida por 64 estudiantes de la Universidad de La Laguna que cursan medicina, 25 hombres (39,06\%) y 39 mujeres (60,93\%), cuyas edades se concentran en el intervalo de 23 a 25 años $(87,5 \%$ ) y que, han realizado la rotación durante las clases no presenciales en "psiquiatría, pediatría y urgencias" (18,8\%), en "psiquiatría y especialidades médicas" (17,2\%), en "ginecología, obstetricia y especialidades quirúrgicas" (9,4\%), y en "pediatría y medicina de familia" (7,8\%).

Tabla 1

Distribución porcentual sobre la rotación realizada

\begin{tabular}{l|c|c|c|c}
\hline Rotaciones & Frecuencia & Porcentaje & $\begin{array}{c}\text { Porcentaje } \\
\text { válido }\end{array}$ & $\begin{array}{c}\text { Porcentaje } \\
\text { acumulado }\end{array}$ \\
\hline $\begin{array}{l}\text { Psiquiatría y } \\
\text { Especialidades Médicas }\end{array}$ & 11 & 17,2 & 17,2 & 17,2 \\
$\begin{array}{l}\text { Especialidades Médicas } \\
\text { Ginecología - Obstetricia y }\end{array}$ & 1 & 1,6 & 1,6 & 18,8 \\
$\begin{array}{l}\text { Especialidades } \\
\text { Quirúrgicas } \\
\text { Ginecología - Obstetricia y } \\
\text { Medicina de Familia }\end{array}$ & 6 & 9,4 & 9,4 & 28,1 \\
\end{tabular}




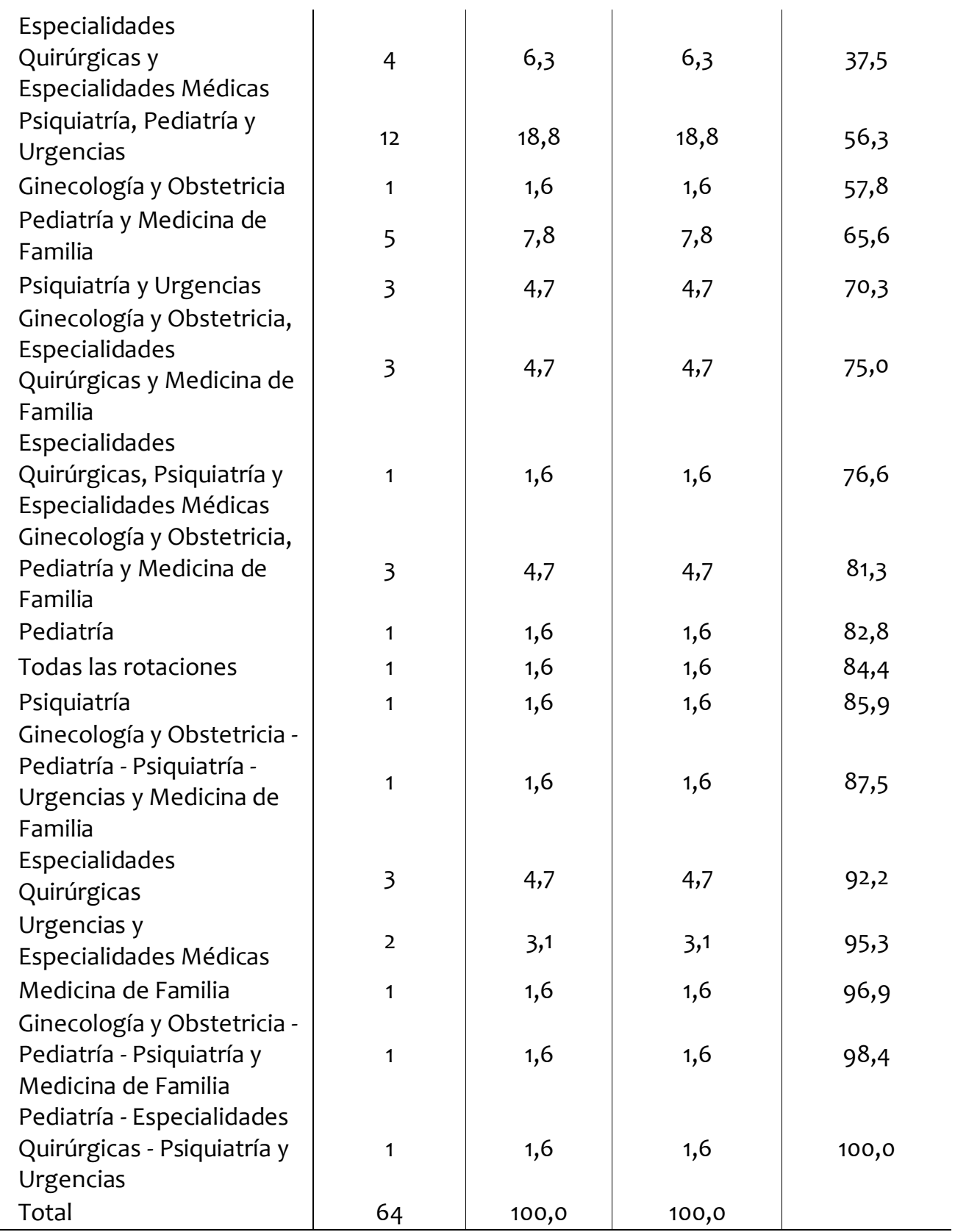

\section{Instrumento y procedimiento}

Aplicamos un cuestionario adaptado a los intereses que perseguimos. Cuestionario de Satisfacción de Alumnos universitarios hacia la formación online (CSAUF), de MonsalveGómez et al. (2014), y el Cuestionario de la Satisfacción de los estudiantes en red en la formación semipresencial (Cabero-Almenara, 2010). Se recogen un conjunto de 46 ítems. Del ítem 1 al ítem 39 se refieren al grado de satisfacción de los estudiantes respecto a criterios pedagógicos-didácticos y del ítem 40 al 46 al nivel de satisfacción con la materia. Los ítems son politómicos y se valoran en una escala tipo Likert de siete opciones de respuesta.

Se estudia la validez del cuestionario aplicado, su consistencia interna y su estructura factorial. Sometemos las variables a estudio para comprobar la pertinencia y viabilidad de que puedan ser factorizadas. Se realiza un Análisis Factorial Exploratorio (AFE) a través del 
método de Componentes Principales (ACP) para verificar la estructura factorial, así como, la fiabilidad del instrumento. Con el ACP se consigue reducir e identificar un número y composición de componentes necesarios (factores) que resumen las puntuaciones del conjunto de variables observadas (Lloret-Segura et al., 2014, p. 1153).

Identificada la estructura factorial y las dimensiones del cuestionario, se realizan análisis univariados (descriptivos), los cuales, resumen las características del conjunto de datos, obteniendo una visión general de la muestra. También las dimensiones se someten a análisis de diferencias de medias mediante las pruebas de Anova y "T" de Levene en función del género, la edad y las rotaciones realizadas. Los diferentes análisis fueron realizados mediante los programas estadísticos IBM SPSS Amos 20.0.

\section{Resultados}

Las valoraciones que realizan los estudiantes respecto al nivel de satisfacción de las materias, las podemos observar en la tabla 2. Para una mejor lectura de los resultados obtenidos, hemos agrupado los valores porcentuales de las alternativas de respuesta "Bastante Insatisfecho y Muy Insatisfecho" cuando refieren a niveles de satisfacción desfavorables, y los de las opciones "Bastante Satisfecho y Muy Satisfecho" cuando refieren a niveles de satisfacción óptimos.

Tabla 2

Nivel de satisfacción de los estudiantes con las materias

\begin{tabular}{l|cccc}
\hline Materias & $\begin{array}{c}\text { \% satisfacción } \\
\text { desfavorable }\end{array}$ & $\begin{array}{c}\text { \% satisfacción } \\
\text { favorable }^{2}\end{array}$ & $\begin{array}{c}\% \\
\text { Total }\end{array}$ & $\mathrm{N}$ \\
\hline Ginecología y Obstetricia & $3,2 \%$ & $32,8 \%$ & $36 \%$ & 56 \\
Especialidades Médicas & $3,2 \%$ & $32,8 \%$ & $36 \%$ & 54 \\
Medicina de Familia & $1,6 \%$ & $28,1 \%$ & $29,7 \%$ & 53 \\
Pediatría & $11 \%$ & $20,4 \%$ & $31,4 \%$ & 54 \\
Especialidades Quirúrgicas & $3,2 \%$ & $28,1 \%$ & $31,3 \%$ & 55 \\
Psiquiatría & $1,6 \%$ & $43,7 \%$ & $45,3 \%$ & 52 \\
Urgencias & $11 \%$ & $12,5 \%$ & $23,5 \%$ & 53 \\
\hline
\end{tabular}

1: Nivel de Satisfacción desfavorable: "Bastante Insatisfecho" y "Muy Insatisfecho"

2: Nivel de Satisfacción favorable: "Bastante Satisfecho" y "Muy Satisfecho"

La materia donde se concentran los porcentajes con mayores niveles de satisfacción es la de Psiquiatría (43,7\%), a la que le siguen Ginecología y Obstetricia y Especialidades Médicas ( $32,8 \%$ ); seguida de las materias de Medicina de Familia y Especialidades Quirúrgicas (28,1\%). Valores no tan positivos nos encontramos con las materias de Urgencias y Pediatría. En la primera, el $11 \%$ de los estudiantes muestra un grado de satisfacción desfavorable; y, en la segunda el $11 \%$ se posiciona en un nivel de satisfacción negativo.

Los índices de bondad de ajuste del modelo para que la matriz de correlaciones de las variables pueda ser factorizada muestran la pertinencia y posibilidad de que el AFE se puede realizar: 
a) Las variables son cuantitativas, continuas, de intervalo y se distribuyen normalmente.

b) El indicador de la prueba de esfericidad de Bartlett presenta una significación de 0,000 , menor a 0,05 .

c) Las correlaciones son altas entre varios grupos de variables, como refleja el determinante de la matriz, con una puntuación distinta al valor 1, Determinante $=7,42 \mathrm{E}-028$.

d) La prueba KMO refleja una puntuación de 0,743 , valor próximo a 1, señal que la matriz de correlaciones puede ser factorizada.

e) Las puntuaciones obtenidas en la diagonal principal de la matriz de correlaciones anti imagen se encuentran por encima de 0,7 , valores cercano a 1 , indicativo que la matriz puede ser factorizada.

Coeficientes y pruebas confirman la pertinencia y condición de que la matriz de correlaciones de las variables pueden ser factorizadas y, por tanto, la posibilidad de llevar a cabo el AFE.

El AFE, mediante el método de componentes principales, extrae una estructura de 5 factores que explican el $80,83 \%$ de la varianza, un porcentaje bastante satisfactorio. Para confirmar estas dimensiones, se procedió a rotar los factores extraídos, siendo la estructura factorial final sobre la satisfacción del alumnado la que sigue:

F1: Formación y la Metodología Docente (58,669\% de la varianza).

F2: Elementos curriculares del Proyecto Docente (6,507\% de la varianza).

F3. Perfil del Docente $(6,265 \%$ de la varianza).

F4. Recursos Didácticos del entorno virtual (5,665\% de la varianza).

F5. Uso y Funcionamiento del entorno virtual (3,728\% de la varianza).

En la tabla 3 figura la estructura factorial final, en la que se describen los ítems que conforman cada factor, sus pesos factoriales, los porcentajes que explican la varianza de cada componente y su consistencia interna.

Tabla 3

Matriz de factores rotados. Estructura factorial final, pesos factoriales, varianza explicada y fiabilidad

\begin{tabular}{|c|c|c|c|c|c|}
\hline Ítems & F1 & F2 & $F_{3}$ & F4 & F5 \\
\hline 3. Utilización de los diferentes recursos on-line & ,832 & & & & \\
\hline 7. Normas de funcionamiento del profesor-tutor & ,804 & & & & \\
\hline 1. Programa de las diferentes rotaciones & ,719 & & & & \\
\hline 14. Originalidad de los contenidos ofrecidos & ,711 & & & & \\
\hline 4. Dominio de la materia del profesor & ,696 & & & & \\
\hline 8. Recomendaciones aportadas por el profesor & ,687 & & & & \\
\hline $\begin{array}{l}\text { 9. Animación y estimulación del profesor tutor sobre la } \\
\text { participación }\end{array}$ & 669 & & & & \\
\hline 2. Aclaraciones aportadas por el profesor & ,653 & & & & \\
\hline 5. Satisfacción con el profesor-tutor & ,635 & & & & \\
\hline 34. Pruebas de evaluación continua y su utilidad & ,600 & & & & \\
\hline $\begin{array}{l}\text { 10.Actividades para facilitar el conocimiento entre el } \\
\text { alumnado }\end{array}$ & ,497 & & & & \\
\hline 6. Calificaciones aportadas en las actividades & , 475 & & & & \\
\hline 13.Comprensión de los contenidos & & ,774 & & & \\
\hline $\begin{array}{l}\text { 15. Interés de los contenidos desde un punto de vista } \\
\text { teórico }\end{array}$ & & ,721 & & & \\
\hline 37. Calidad y explicación de los materiales docentes & & ,632 & & & \\
\hline
\end{tabular}


16. Interés de los contenidos desde un punto de vista práctico

, 598

33. Utilidad de los materiales aportados por el docente

35. Ponderación y rúbricas de los procedimientos de evaluación

17. Contenidos agradables

12. Volumen de información de los contenidos

18. Relación entre los objetivos y los contenidos

36. Porcentaje asignado a la evaluación sobre la participación

20. Calidad científica y didáctica de los contenidos

39. Carga de trabajo de la asignatura

19. Periodo de tiempo destinado para los contenidos

21. Comunicación con los profesores-tutores

32. Accesibilidad docente en las horas de tutoría y fuera de clase

22. Comunicación con el resto de los compañeros

31. Interés del profesor hacia la asignatura

11. Diferentes contenidos que se presentan

38. Motivación del profesor a la participación del alumno

26. Adecuación entre los diferentes elementos estéticos

25. Calidad estética del entorno

30.Utilización de los materiales didácticos docentes

28. Casos clínicos aportados en los talleres

23. Funcionamiento técnico del entorno

24. Recursos didácticos utilizados

27. Tiempos de respuesta de la plataforma

29. Interés por la asignatura

\section{\% Varianza}

a de Cronbach
, 548

, 546

, 532

,521

, 519

,497

, 482

,445

,409

, 724

,665

,655

,629

, 549

,522

,864

, 741

,651

,461

Las saturaciones de los factores generados oscilan entre $\alpha=0,842$ y $\alpha=0,967$ : a) F1: Satisfacción del alumnado con la Formación y la Metodología Docente $(a=, 967)$; b) F2: Satisfacción del alumnado con los Elementos curriculares del Proyecto Docente ( $\alpha=, 955)$; c) F3: Satisfacción del alumnado con el Perfil del Docente $(\alpha=, 900)$; d) F4: Satisfacción del alumnado con los Recursos Didácticos del entorno virtual $(\alpha=, 866)$; e) F5: Satisfacción del alumnado con el Uso y Funcionamiento del entorno virtual $(\alpha=, 842)$. El Cuestionario goza de una alta confiabilidad, $\alpha=0,979$, un coeficiente excelente.

Los análisis efectuados confirman la solidez del cuestionario. Presenta una estructura factorial parsimoniosa, validez de constructo y una alta fiabilidad, tanto a nivel global como al de las subescalas.

\section{Análisis descriptivo del cuestionario de satisfacción de la formación no presencial}

La valoración respecto al nivel de satisfacción, en conjunto, es bastante favorable, según las puntuaciones medias obtenidas en cada una de las dimensiones, las cuales indican que se posicionan entre las opciones de respuesta "bastante y muy satisfecho". La experiencia para los estudiantes ha sido favorable. De forma más específica, las siguientes tablas reflejan tanto las puntuaciones medias como las frecuencias obtenidas en cada uno de los ítems que conforman las 5 dimensiones. Con respecto a los valores porcentuales de las opciones de respuestas emitidas por los estudiantes, para una mejor lectura e interpretación, se han agrupado en dos grupos: el primero refiere a niveles de satisfacción desfavorables cuando las 
alternativas de respuesta se posicionan en "Bastante Insatisfecho y Muy Insatisfecho" y, la segunda, refiere a niveles de satisfacción óptimos cuando las opciones de respuesta se posicionan en "Bastante Satisfecho y Muy Satisfecho".

Tabla 4

Descriptivos: Puntuaciones medias y Frecuencias de la Dimensión Satisfacción del alumnado con la Formación y la Metodología Docente

\begin{tabular}{|c|c|c|c|c|}
\hline Ítems & Media $^{(a)}$ & $\begin{array}{c}\text { \% Nivel de } \\
\text { satisfacción } \\
\text { desfavorable }^{(b)}\end{array}$ & $\begin{array}{c}\text { \% Nivel de } \\
\text { satisfacción } \\
\text { favorable }^{(c)}\end{array}$ & $\mathrm{N}$ \\
\hline 1.Programa de las diferentes rotaciones & 5,24 & $4,7 \%$ & $54,7 \%$ & 63 \\
\hline 2.Aclaraciones aportadas por el profesor & 4,97 & $14,1 \%$ & $46,9 \%$ & 63 \\
\hline 3. Utilización de los diferentes recursos & 4,91 & $10,9 \%$ & $46,8 \%$ & 64 \\
\hline 4. Dominio de la materia del profesor-tutor & 5,25 & $6,2 \%$ & $54,7 \%$ & 64 \\
\hline 5. La información de los contenidos & 4,75 & $7,8 \%$ & $37,5 \%$ & 63 \\
\hline 6. Calificaciones de las actividades & 4,89 & $7,8 \%$ & $37,5 \%$ & 63 \\
\hline 7.Explicación de las normas de funcionamiento & 4,92 & $9,4 \%$ & $45,3 \%$ & 64 \\
\hline 8. Recomendaciones aportadas para el trabajo & 5,08 & $4,7 \%$ & $43,7 \%$ & 64 \\
\hline $\begin{array}{l}\text { 9. Animación y estimulación del profesor tutor } \\
\text { a la participación }\end{array}$ & 4,58 & $15,6 \%$ & $39 \%$ & 64 \\
\hline $\begin{array}{l}\text { 10. Actividades para el conocimiento entre el } \\
\text { alumnado }\end{array}$ & 4,69 & $12,5 \%$ & $39,1 \%$ & 64 \\
\hline 14. Originalidad de los contenidos & 4,94 & $12,5 \%$ & $45,3 \%$ & 64 \\
\hline 34. Pruebas de evaluación continua y su utilidad & 4,89 & $14,1 \%$ & $45,3 \%$ & 64 \\
\hline
\end{tabular}

(a): Escala de valores: "1" Muy insatisfecho; "2" Bastante insatisfecho; "3" Algo insatisfecho; "4" Indiferente;

"5" Algo satisfecho; "6" Bastante satisfecho; "7" Muy satisfecho

(b): Nivel de Satisfacción desfavorable: "Bastante Insatisfecho" y "Muy Insatisfecho"

(c): Nivel de Satisfacción favorable: "Bastante Satisfecho" y "Muy Satisfecho"

En la tabla 5, los ítems son valorados de manera positiva. Destacan el ítem 1 y el 4, con una $7=$ 5,24 y un valor porcentual del $54,7 \%$ en el caso del primero y una $0=5,25$ e idéntico porcentaje que su predecesor en el caso del segundo. Más de la mitad de la muestra manifiesta un nivel de satisfacción favorable. Le siguen la de las preguntas 2, 3, 7, 8, 14 y 15, con porcentajes superiores al $40 \%$ respecto al grado de satisfacción positivo que declaran tener los estudiantes. La media global de esta dimensión se sitúa en un $\mathbf{7}=4,93$.

Tabla 5

Descriptivos: Puntuaciones medias y Frecuencias de la Dimensión Satisfacción del alumnado

\begin{tabular}{|c|c|c|c|c|}
\hline Ítems & Media $^{(a)}$ & $\begin{array}{c}\text { \% Nivel de } \\
\text { satisfacción } \\
\text { desfavorable }^{(b)}\end{array}$ & $\begin{array}{c}\text { \% Nivel de } \\
\text { satisfacción } \\
\text { favorable }\end{array}$ & $\mathrm{N}$ \\
\hline 12. Volumen de información para la formación & 4,98 & $11 \%$ & $51,6 \%$ & 64 \\
\hline 13. Contenidos presentados fáciles de comprender & 5,38 & $3,1 \%$ & $46,3 \%$ & 64 \\
\hline $\begin{array}{l}\text { 15. Interés de los contenidos desde un punto de } \\
\text { vista teórico }\end{array}$ & 5,33 & $3,1 \%$ & $53,2 \%$ & 64 \\
\hline $\begin{array}{l}\text { 16. Interés de los contenidos desde un punto de } \\
\text { vista práctico }\end{array}$ & 5,00 & $12,5 \%$ & $45,3 \%$ & 64 \\
\hline
\end{tabular}




\begin{tabular}{l|cccc} 
17. Contenidos son agradables & $\mathbf{5 , 1 9}$ & $\mathbf{6 , 2} \%$ & $\mathbf{5 4 , 7 \%}$ & 64 \\
18. Relación entre los objetivos y los contenidos & $\mathbf{5 , 0 8}$ & $\mathbf{7 , 8}$ & $\mathbf{5 0 \%}$ & 64 \\
19. Periodo de tiempo destinado contenidos & $\mathbf{5 , 3 4}$ & $\mathbf{9 , 4} \%$ & $\mathbf{6 4 \%}$ & 64 \\
20. Calidad científica y didáctica de los contenidos & $\mathbf{5 , 2 0}$ & $\mathbf{4 , 7}$ & $\mathbf{5 4 , 7 \%}$ & 64 \\
33. Utilidad de los materiales docente & $\mathbf{5 , 0 2}$ & $\mathbf{9 , 4} \%$ & $\mathbf{5 1 , 6 \%}$ & 64 \\
35. Ponderación rúbricas en la evaluación & 4,84 & $9,4 \%$ & $33,7 \%$ & 62 \\
36.Porcentaje asignado a la participación & 4,87 & $\mathbf{7 , 8}$ & $42,2 \%$ & 63 \\
37. Calidad y explicación de los materiales docentes & $\mathbf{4 , 8 8}$ & $\mathbf{1 4 , 1 \%}$ & $\mathbf{4 6 , 9 \%}$ & 64 \\
39. Carga de trabajo de la asignatura & $\mathbf{5 , 1 1}$ & $\mathbf{9 , 4} \%$ & $\mathbf{5 1 , 6 \%}$ & 63
\end{tabular}

(a): Escala de valores: "1" Muy insatisfecho; "2" Bastante insatisfecho; "3" Algo insatisfecho; "4" Indiferente;

"5" Algo satisfecho; "6" Bastante satisfecho; "7" Muy satisfecho

(b): Nivel de Satisfacción desfavorable: "Bastante Insatisfecho" y "Muy Insatisfecho"

(c): Nivel de Satisfacción favorable: "Bastante Satisfecho" y "Muy Satisfecho"

Del mismo modo que sucediera en la dimensión precedente, los valores obtenidos, tanto en las puntuaciones medias como en los porcentajes de los niveles de satisfacción favorables, son altos. Sobresale el ítem 19, con un 64\% de respuestas, que los estudiantes están bastantes y muy satisfechos con el contenido de esta pregunta: "el periodo de tiempo destinado para el desarrollo de los contenidos". Los valores porcentuales de los niveles de satisfacción favorables, en la mayoría de los ítems de esta dimensión, superan el $50 \%$ de respuestas positivas. La media global de esta dimensión es de ? =5,09.

Tabla 6

Descriptivos: Puntuaciones medias y Frecuencias de los ítems de la Dimensión Satisfacción del alumnado con el Perfil del Docente

\begin{tabular}{|c|c|c|c|c|}
\hline Ítems & Media $^{(a)}$ & $\begin{array}{c}\text { \% Nivel de } \\
\text { satisfacción } \\
\text { desfavorable }\end{array}$ & $\begin{array}{c}\text { \% Nivel de } \\
\text { satisfacción } \\
\text { favorable }^{(c)}\end{array}$ & $\mathrm{N}$ \\
\hline 11. Contenidos actuales & 5,78 & $1,6 \%$ & $73,4 \%$ & 64 \\
\hline $\begin{array}{l}\text { 21.Comunicación con los profesores-tutores } \\
\text { mediante las herramientas de comunicación }\end{array}$ & 5,55 & $7,8 \%$ & $64,1 \%$ & 64 \\
\hline 22. Comunicación on-line con los compañeros & 6,17 & $1,6 \%$ & $81,2 \%$ & 64 \\
\hline 31. Interés del profesor hacia la asignatura & 5,45 & $7,8 \%$ & $61 \%$ & 64 \\
\hline $\begin{array}{l}\text { 32. Accesibilidad del profesor en las horas de } \\
\text { tutoría y fuera de clase }\end{array}$ & 5,36 & $4,7 \%$ & $62,5 \%$ & 64 \\
\hline $\begin{array}{l}\text { 38. Motivación por parte del profesor a la } \\
\text { participación }\end{array}$ & 5,05 & $7,8 \%$ & $51,6 \%$ & 64 \\
\hline
\end{tabular}

(a): Escala de valores: "1" Muy insatisfecho; "2" Bastante insatisfecho; "3" Algo insatisfecho; "4" Indiferente;

"5" Algo satisfecho; "6" Bastante satisfecho; "7" Muy satisfecho

(b): Nivel de Satisfacción desfavorable: "Bastante Insatisfecho" y "Muy Insatisfecho"

(c): Nivel de Satisfacción favorable: "Bastante Satisfecho" y "Muy Satisfecho"

Datos de la tabla 6 no dejan lugar a dudas. Dimensión muy bien considerada por los estudiantes. La puntuación media global de la dimensión es ?= 5,56 y las frecuencias alcanzadas, en los niveles de satisfacción favorables ( 51,6\% y el 81,2\%). Señalar los ítems 22,11 y 21. 
Tabla 7

Descriptivos: Puntuaciones medias Frecuencias de los ítems de la Dimensión Satisfacción del alumnado con los Recursos Didácticos del entorno virtual

\begin{tabular}{|c|c|c|c|c|}
\hline Ítems & Media $^{(a)}$ & $\begin{array}{c}\text { \% Nivel de } \\
\text { satisfacción } \\
\text { desfavorable } \\
\text { (b) }\end{array}$ & $\begin{array}{l}\text { \% Nivel de } \\
\text { satisfacción } \\
\text { favorable }^{(c)}\end{array}$ & $\mathrm{N}$ \\
\hline 25. Calidad estética del entorno & 5,08 & $7,8 \%$ & $51,6 \%$ & 64 \\
\hline $\begin{array}{l}\text { 26. Adecuación entre los diferentes elementos } \\
\text { estéticos }\end{array}$ & 5,08 & $3,2 \%$ & $46,9 \%$ & 64 \\
\hline 28. Casos clínicos aportados en los talleres & 5,46 & $1,6 \%$ & $56,3 \%$ & 63 \\
\hline $\begin{array}{l}\text { 30.Utilización de los materiales didácticos } \\
\text { docentes }\end{array}$ & 5,36 & $0,0 \%$ & $62,5 \%$ & 64 \\
\hline
\end{tabular}

(a): Escala de valores: "1" Muy insatisfecho; "2" Bastante insatisfecho; "3" Algo insatisfecho; "4" Indiferente

" 5 " Algo satisfecho; "6" Bastante satisfecho; "7" Muy satisfecho

(b): Nivel de Satisfacción desfavorable: "Bastante Insatisfecho" y "Muy Insatisfecho"

(c): Nivel de Satisfacción favorable: "Bastante Satisfecho" y "Muy Satisfecho"

Las preguntas son valoradas de manera positiva, destacando los ítems 30 y 28. La puntuación media global de esta dimensión es de $0=5,24$. La tabla 8 revela las puntuaciones de los ítems de la dimensión Satisfacción del alumnado con el Uso y Funcionamiento del entorno virtual.

Tabla 8

Descriptivos: Puntuaciones medias y Frecuencias de los ítems de la Dimensión Satisfacción del alumnado con el Uso y Funcionamiento del entorno virtual

\begin{tabular}{|c|c|c|c|c|}
\hline Ítems & Media $^{(a)}$ & $\begin{array}{c}\text { \% Nivel de } \\
\text { satisfacción } \\
\text { desfavorable }\end{array}$ & $\begin{array}{c}\text { \% Nivel de } \\
\text { satisfacción } \\
\text { favorable }^{(c)}\end{array}$ & $\mathrm{N}$ \\
\hline 23. Funcionamiento técnico del entorno & 6,11 & $0,0 \%$ & $78,1 \%$ & 64 \\
\hline 24. Recursos didácticos utilizados & 5,52 & $4,7 \%$ & $60,9 \%$ & 64 \\
\hline 27. Tiempos de respuesta de la plataforma & 5,48 & $3,1 \%$ & $62,5 \%$ & 64 \\
\hline 29. Interés por la asignatura & 5,72 & $1,6 \%$ & $70,4 \%$ & 64 \\
\hline
\end{tabular}

(a): Escala de valores: "1" Muy insatisfecho; " 2 " Bastante insatisfecho; " 3 " Algo insatisfecho; " 4 " Indiferente;

"5" Algo satisfecho; "6" Bastante satisfecho; "7" Muy satisfecho

(b): Nivel de Satisfacción desfavorable: "Bastante Insatisfecho" y "Muy Insatisfecho"

(c): Nivel de Satisfacción favorable: "Bastante Satisfecho" y "Muy Satisfecho"

Dimensión que presenta la puntuación media global más alta, $1=5,70$, que se refleja en los valores porcentuales obtenidos en relación con los niveles de satisfacción favorables (60\%). Con respecto a la existencia de diferencias estadísticamente significativas en las variables criteriales o independientes en función del género, de la edad y de las rotaciones realizadas, hemos encontrado los siguientes hallazgos: 


\section{a) Género}

\section{Tabla 9}

Prueba T de Levene para muestras independientes

\begin{tabular}{|c|c|c|c|c|c|c|c|c|}
\hline \multirow{3}{*}{\multicolumn{2}{|c|}{ Dimensiones }} & \multicolumn{2}{|c|}{$\begin{array}{c}\text { Prueba de Levene } \\
\text { igualdad de } \\
\text { varianzas }\end{array}$} & \multicolumn{5}{|c|}{ Prueba T igualdad de medias } \\
\hline & & \multirow[t]{2}{*}{$\mathrm{F}$} & \multirow[t]{2}{*}{ Sig. } & \multirow[t]{2}{*}{$\mathrm{T}$} & \multirow[t]{2}{*}{$\mathrm{Gl}$} & \multirow{2}{*}{$\begin{array}{c}\text { Sig. } \\
\text { (bilateral) }\end{array}$} & \multicolumn{2}{|c|}{$\begin{array}{c}95 \% \text { Intervalo de } \\
\text { confianza para la } \\
\text { diferencia }\end{array}$} \\
\hline & & & & & & & Superior & Inferior \\
\hline & $\begin{array}{l}\text { Se han asumido } \\
\text { varianzas } \\
\text { iguales }\end{array}$ & 133 & ,717 & ,425 & 57 & 672 & $\begin{array}{c}- \\
, 4276378 \\
1\end{array}$ & ,65815784 \\
\hline & $\begin{array}{l}\text { No se han } \\
\text { asumido } \\
\text { varianzas } \\
\text { iguales }\end{array}$ & & & ,419 & 42,254 & 677 & $\begin{array}{c}- \\
, 439899 \\
80\end{array}$ & 67041984 \\
\hline \multirow[t]{2}{*}{$\mathrm{D} 2 *$} & $\begin{array}{l}\text { Se han asumido } \\
\text { varianzas } \\
\text { iguales }\end{array}$ & 1,216 & ,275 &,- 487 & 57 & ,628 & $\begin{array}{c}- \\
, 6773006 \\
7\end{array}$ & ,41223360 \\
\hline & $\begin{array}{l}\text { No se han } \\
\text { asumido } \\
\text { varianzas } \\
\text { iguales }\end{array}$ & & &,- 455 & 35,572 & 652 & $\begin{array}{c}- \\
, 7236243 \\
5\end{array}$ & , 45855729 \\
\hline \multirow[t]{2}{*}{$\mathrm{D}_{3}{ }^{*}$} & $\begin{array}{l}\text { Se han asumido } \\
\text { varianzas } \\
\text { iouales }\end{array}$ & ,334 &, 566 & $-1,085$ & 57 & ,283 & $\begin{array}{c}- \\
, 8362351\end{array}$ & $\begin{array}{c}, 2486649 \\
9\end{array}$ \\
\hline & $\begin{array}{l}\text { No se han } \\
\text { asumido } \\
\text { varianzas } \\
\text { iguales }\end{array}$ & & & $-1,206$ & 56,502 & ,233 & $\begin{array}{c}6 \\
- \\
, 7817860 \\
9\end{array}$ & ,19421593 \\
\hline \multirow[t]{2}{*}{$\mathrm{D} 4 *$} & $\begin{array}{l}\text { Se han asumido } \\
\text { varianzas } \\
\text { iguales }\end{array}$ & 692 & 409 & $-1,281$ & 57 & ,205 & $\begin{array}{c}8863437 \\
1\end{array}$ & ,19470175 \\
\hline & $\begin{array}{l}\text { No se han } \\
\text { asumido } \\
\text { varianzas } \\
\text { iguales }\end{array}$ & & & $-1,354$ & 51,836 & ,181 & $\begin{array}{c}- \\
, 8581834 \\
6\end{array}$ & , 16654150 \\
\hline \multirow[t]{2}{*}{$\mathrm{D}_{5}^{*}$} & $\begin{array}{l}\text { Se han asumido } \\
\text { varianzas } \\
\text { iguales }\end{array}$ & , 001 & ,975 & 380 & 57 & ,705 & $\begin{array}{c}4438616 \\
3\end{array}$ & ,65165459 \\
\hline & $\begin{array}{l}\text { No se han } \\
\text { asumido } \\
\text { varianzas } \\
\text { iguales }\end{array}$ & & & 374 & 42,301 & ,710 & $\begin{array}{c}- \\
4450190 \\
4\end{array}$ & ,66381200 \\
\hline
\end{tabular}

* D1= Satisfacción del alumnado con la Formación y la Metodología Docente; D2= Satisfacción del alumnado con los Elementos curriculares del Proyecto Docente; D3= Satisfacción del alumnado con el Perfil del Docente; D4= Satisfacción del alumnado con los Recursos Didácticos del entorno virtual; D5= Satisfacción del alumnado con el Uso y Funcionamiento del entorno virtual

De la lectura de la tabla 9 podemos ver que " $\mathrm{t}$ " > 0,05 en todas las dimensiones, lo que se traduce que no se rechaza, sino que se acepta la hipótesis nula $\left(\mathrm{H}_{0}\right)$ ante la ausencia de diferencias entre ambos sexos. Por tanto, no existen diferencias estadísticamente significativas en las diferentes dimensiones por razón del género. 
b) Edad

Tabla 10

Prueba de Anova intergrupos por razón de la edad

\begin{tabular}{|c|c|c|c|c|c|c|}
\hline & & $\begin{array}{c}\text { Suma de } \\
\text { cuadrados }\end{array}$ & Gl & $\begin{array}{c}\text { Media } \\
\text { cuadrática }\end{array}$ & $\mathrm{F}$ & Sig. \\
\hline \multirow[t]{3}{*}{ D1 * EDAD } & Inter-grupos & 1,647 & 2 & ,823 & ,818 & ,446 \\
\hline & Intra-grupos & 57,353 & 57 & 1,006 & & \\
\hline & Total & 59,000 & 59 & & & \\
\hline \multirow[t]{3}{*}{$\mathrm{D} 2$ * EDAD } & Inter-grupos & 3,285 & 2 & 1,642 & 1,680 & 195 \\
\hline & Intra-grupos & 55,715 & 57 & ,977 & & \\
\hline & Total & 59,000 & 59 & & & \\
\hline \multirow[t]{3}{*}{ D3 * EDAD } & Inter-grupos & 1,290 & 2 & ,645 & 637 & ,533 \\
\hline & Intra-grupos & 57,710 & 57 & 1,012 & & \\
\hline & Total & 59,000 & 59 & & & \\
\hline \multirow[t]{3}{*}{$\mathrm{D} 4$ * EDAD } & Inter-grupos & 2,180 & 2 & 1,090 & 1,094 &, 342 \\
\hline & Intra-grupos & 56,820 & 57 & ,997 & & \\
\hline & Total & 59,000 & 59 & & & \\
\hline \multirow[t]{3}{*}{ D5 * EDAD } & Inter-grupos & 1,936 & 2 & ,968 & ,967 &, 386 \\
\hline & Intra-grupos & 57,064 & 57 & 1,001 & & \\
\hline & Total & 59,000 & 59 & & & \\
\hline
\end{tabular}

* D1= Satisfacción del alumnado con la Formación y la Metodología Docente; D2= Satisfacción del alumnado con los Elementos curriculares del Proyecto Docente; D3= Satisfacción del alumnado con el Perfil del Docente; D4= Satisfacción del alumnado con los Recursos Didácticos del entorno virtual; D5= Satisfacción del alumnado con el Uso y Funcionamiento del entorno virtual

Del mismo modo que sucediera en el análisis anterior, de la tabla 10 podemos ver que "p"> 0,05 en todas las dimensiones, lo que significa que no se rechaza, sino que ya que no se encuentran diferencias entre los grupos de edad. No existen diferencias estadísticamente significativas en las diferentes dimensiones por razón de la edad.

\section{c) Rotaciones realizadas}

Tampoco se hallan diferencias (tabla 11) puesto que "p"> 0,05 en todas las dimensiones; no se rechaza, sino que se acepta la hipótesis nula $\left(\mathrm{H}_{0}\right)$ ya que, no se encuentran diferencias entre las rotaciones practicadas por los estudiantes durante las clases no presenciales. No existen diferencias estadísticamente significativas en las diferentes dimensiones por razón de las rotaciones realizadas.

\section{Tabla 11}

Prueba de Anova intergrupos por razón de las rotaciones

\begin{tabular}{|c|c|c|c|c|c|c|}
\hline & & $\begin{array}{c}\text { Suma de } \\
\text { cuadrados }\end{array}$ & $\mathrm{Gl}$ & $\begin{array}{c}\text { Media } \\
\text { cuadrática }\end{array}$ & $\mathrm{F}$ & Sig. \\
\hline \multirow{3}{*}{$\begin{array}{l}\text { D1* } \\
\text { ROTACIONES }\end{array}$} & Inter-grupos & 25,056 & 20 & 1,253 & 1,439 & ,162 \\
\hline & \multirow{2}{*}{$\begin{array}{l}\text { Intra-grupos } \\
\text { Total }\end{array}$} & 33,944 & 39 & ,870 & & \\
\hline & & 59,000 & 59 & & & \\
\hline \multirow{3}{*}{$\begin{array}{l}\text { D2 * } \\
\text { ROTACIONES }\end{array}$} & Inter-grupos & 13,850 & 20 & 693 & ,598 & 890 \\
\hline & \multirow{2}{*}{$\begin{array}{l}\text { Intra-grupos } \\
\text { Total }\end{array}$} & 45,150 & 39 & 1,158 & & \\
\hline & & 59,000 & 59 & & & \\
\hline \multirow{3}{*}{$\begin{array}{l}\text { D3 * } \\
\text { ROTACIONES }\end{array}$} & Inter-grupos & 21,094 & 20 & 1,055 & 1,085 & 401 \\
\hline & Intra-grupos & 37,906 & 39 & ,972 & & \\
\hline & Total & 59,000 & 59 & & & \\
\hline
\end{tabular}




\begin{tabular}{|c|c|c|c|c|c|c|}
\hline $\mathrm{D}_{4}$ * & Inter-grupos & 21,196 & 20 & 1,060 & 1,093 & ,393 \\
\hline \multirow[t]{2}{*}{ ROTACIONES } & \multirow{2}{*}{$\begin{array}{l}\text { Intra-grupos } \\
\text { Total }\end{array}$} & 37,804 & 39 & ,969 & \multirow{5}{*}{1,238} & \multirow{5}{*}{,277 } \\
\hline & & 59,000 & 59 & & & \\
\hline \multirow{3}{*}{$\begin{array}{l}\text { D5 * } \\
\text { ROTACIONES }\end{array}$} & Inter-grupos & 22,914 & 20 & 1,146 & & \\
\hline & Intra-grupos & 36,086 & 39 & ,925 & & \\
\hline & Total & 59,000 & 59 & & & \\
\hline
\end{tabular}

* D1= Satisfacción del alumnado con la Formación y la Metodología Docente; D2= Satisfacción del alumnado con los Elementos curriculares del Proyecto Docente; D3= Satisfacción del alumnado con el Perfil del Docente; D4= Satisfacción del alumnado con los Recursos Didácticos del entorno virtual; D5= Satisfacción del alumnado con el Uso y Funcionamiento del entorno virtual

\section{Discusión y conclusiones}

El cuestionario revela una satisfactoria calidad métrica, metodológica y una alta consistencia interna. Goza de una estructura factorial bien definida y parsimoniosa, de la cual se genera cinco factores con una buena estructura interna y saturaciones factoriales altas que explican el $80,83 \%$ de la varianza y hace que el instrumento sea válido.

Los resultados evidencian que el grado de satisfacción es bastante alto con la formación recibida; pero, además, las distintas dimensiones que estructuran el cuestionario aplicado gozan de un nivel de satisfacción favorable. La experiencia vivida en esta clase de formación es valorada de forma positiva. Puntúan de manera más favorable aquellas dimensiones referidas, en el siguiente orden, a la "Satisfacción con el Uso y Funcionamiento del entorno virtual", la "Satisfacción con el Perfil del Docente" y la "Satisfacción con los Recursos Didácticos del entorno virtual"; "Satisfacción con los Elementos curriculares del Proyecto Docente" y "Satisfacción con la Formación y la Metodología Docente".

Los datos invitan a pensar que el uso, funcionamiento y recursos didácticos que el profesor emplea en el entorno virtual, así como su perfil docente, son aspectos fundamentales que sustentan el proceso de enseñanza-aprendizaje en este tipo de formación no presencial. Compartimos con Cabero-Almenara et al. (2010) la actitud favorable del alumnado hacia la formación semipresencial, así como, la necesidad de la existencia en la institución superior de planes formativos orientados hacia la formación del profesorado en dichas acciones formativas.

No se han encontrado diferencias estadísticamente significativas en las dimensiones por razón del género, edad y rotaciones realizadas durante el curso. No son variables que influyan en la satisfacción de la formación no presencial.

La investigación tiene una limitación en proporción a la muestra. Aconsejable aplicar este Cuestionario mediante muestreo aleatorio estratificado, donde la población universitaria se dividiera en grupos en función de características determinadas para, elegir la muestra aleatoriamente y su proporcionalidad. Este método justificaría la representatividad del muestreo. Después de la pandemia es necesario un replanteamiento sobre la misión de la institución superior, teniendo en cuenta los modelos presenciales, on-line y mixtos (Zerpa y Zerpa, 2020).

Las tecnologías educativas, recobra una gran fortaleza frente a las debilidades formativas de los docentes, lo que implica que, los planes formativos podrían ser, reorientados hacia la 
mejora de la competencia digital. Los marcos competenciales sobre los cuales se forman los docentes deberían enfocarse hacia los obtenidos en el estudio de Cabero-Almenara et al., (2020).

El diseño de los programas docentes, demandan planteamientos más acordes al tipo de enseñanza. Momento para que los planes formativos de la Universidad contemplen acciones formativas orientadas hacia la innovación docente. Entorno virtual y perfil docente, podrían ser los pilares básicos para la formación docente asociados al grado de satisfacción del alumnado por Facultad.

\section{Referencias}

Bunge, M. (2010). Las pseudociencias. Laetoli.

Cabero-Almenara, J., Llorente, C., y Puentes, Á. (2010). La satisfacción de los estudiantes en red en la formación semipresencial. Comunicar, 18(35), 149-157.

Cabero-Almenara, J., Barroso-Osuna, J., Palacios Rodríguez, A., y Llorente-Cejudo, C. (2020). Marcos de Competencias Digitales para docentes universitarios: su evaluación a través del coeficiente competencia experta. Revista Electrónica Interuniversitaria De Formación Del Profesorado, 23 (3), 17-34. https://doi.org/10.6018/reifop.414501.

Cerón, M.C. (2006). Metodologías de la investigación Social. LOM ediciones.

Cochran, W. G., y Bouclier, A. S. (1980). Técnicas de muestreo. Continental.

Cuso, F. J. P., Clares, P. M., y Juárez, M. M. (2015). Satisfacción del estudiante universitario con la tutoría. Diseño y validación de un instrumento de medida. Estudios sobre educación, 29, 81-101.

De Agüero-Servín, M. (2020). De certezas e incertidumbres. La tecnología como una manera de crear y transformar la educación y la sociedad. Revista Digital Universitaria, 21 (3), 1-5. http://doi.org/10.22201/ codeic.16076079e.2020.v21n3.ao.

Hernández R.; Fernández, C. y Baptista, P. (2010). Metodología de la investigación. McGrawHill.

Kerlinger, F.N. (1979). Enfoque conceptual de la investigación del comportamiento. Nueva Editorial Interamericana.

Lloret-Segura, S., Ferreres-Traver, A., Hernández-Baeza, A., y Tomás-Marco, I. (2014). El análisis factorial exploratorio de los ítems: una guía práctica, revisada y actualizada. Anales de psicología, 30(3), 1151-1169.

Melo, B. I., Moreira, L. O., Villalobos, C. P., Araneda, G. T., Calvo, P. M., Kother, A. M. y Durán, C. B. (2015). Estructura factorial y confiabilidad del Cuestionario de Satisfacción Académica en estudiantes de medicina chilenos. Revista Iberoamericana de Diagnóstico y Evaluación-e Avaliação Psicológica, 2(40), 73-82.

Merino-Soto, C., Dominguez-Lara, S., y Fernández-Arata, M. (2017). Validación inicial de una Escala Breve de Satisfacción con los Estudios en estudiantes universitarios de Lima. Educación Médica, 18(1), 74-77. 
Monsalve-Gómez, J. C., Botero-Botero, J. A., y Montoya-Suárez, L. M. (2014). Evaluación de una Experiencia de Formación B-Learning en el Aprendizaje de Tecnologías de la Información y la Comunicación. Lámpsakos, (11), 59-65.

Ordorika, I. (2020). Pandemia y educación superior. Revista de la Educación Superior, 49 (194), $1-8$.

Pineda-Alfonso, J. A., y Piña, O. M. D. (2020). Las concepciones pedagógicas del profesorado universitario: un punto de partida para el cambio docente. Educación XXI, 23 (2), 95118.

Robbins, S. (1998). Comportamiento Organizacional. Prentice Hall.

Toledo, P. A. (2020). Pandemia Covid-19: Educación a Distancia. O las Distancias en la Educación. Revista Internacional de Educación para la Justicia Social, 9 (3), 1-3.

Zerpa, C. A. P., y Zerpa, M. Y. P. (2020). Clases on line con móviles durante la pandemia (estudio de caso). Revista Vinculando. Recuperado de: http://vinculando.org/educacion/clases-on-line-con-moviles-durante-lapandemia-estudio-de-caso.html. 